\title{
THREE-MANIFOLD SUBGROUP GROWTH, HOMOLOGY OF COVERINGS AND SIMPLICIAL VOLUME*
}

\author{
ALEXANDER REZNIKOV ${ }^{\dagger}$ AND PIETER MOREE ${ }^{\ddagger}$
}

1. Introduction. This paper is concerned with the conjecture, communicated to the first author by A. Lubotzky and A. Shalev:

Conjecture 1.1. Let $M$ be a hyperbolic three-manifold. Let $f(d)$ denote the number of subgroups of index $d$ in $\pi_{1}(M)$. There exists an absolute positive constant $C_{1}$ such that, for infinitely many $d, f(d)>\exp \left(C_{1} d\right)$.

This conjecture follows easily from the following one:

Conjecture 1.2. Let $M$ be as above. For any prime $p$ there exists infinitely many $d$, for which there exists a d-sheeted covering $N$ of $M$ such that

$$
\operatorname{rank}_{p}\left(H_{1}(N)\right)>C_{2} d
$$

where $C_{2}$ is an absolute positive constant.

Observe that for any finitely generated group $G$, and a subgroup $H$ of index $d$, $\operatorname{rank}_{p}\left(H_{1}(H)\right) \leq$ const $\cdot d$, so that (1) is sharp up to a constant.

A much weaker growth rate than conjectured in (1), namely, $\operatorname{rank}_{p}\left(H_{1}(N)\right)>$ $(\log d)^{2-\epsilon}$ has been proved by Shalev [Sh]. It follows from the Class Tower Theorem of [R1] that $\operatorname{rank}_{p}\left(H_{1}(N)\right)>(\log d)^{2}$.

These conjectures about the subgroup growth should be compared with the results of [Tu] and [SW] concerning the word growth of $\pi_{1}(M)$.

Here we prove the following result for a priori a much wider class of manifolds than hyperbolic manifolds (given the present status of the hyperbolization conjecture). Recall the definition of rich fundamental groups given in [R1]:

(R) A closed irreducible three-manifold satisfies condition (R) if either

(a) the Casson invariant $\lambda(M)>\sharp\left(\right.$ representations of $\pi_{1}(M)$ in $\left.S L_{2}\left(\mathbb{F}_{5}\right)\right)$ or

(b) $M$ is hyperbolic.

MAIN THEOREM 1.1. Suppose the three-manifold $M$ is a rational homology sphere (that is $H_{1}(M, \mathbb{Q})=0$ ) satisfying $(R)$. Then for all, but at most two, primes $\ell$ with $\ell \equiv 3(\bmod 4)$, there exists a positive $\alpha$ such that for infinitely many $d$, there exists a d-sheeted covering $N$ of $M$ such that either the inequality $\operatorname{rank}_{\ell} H_{1}(N)>c d^{\alpha}$, or $\operatorname{rank}_{\mathbb{Z}} H_{1}(N)>c d^{1 / 3}$, holds.

As a corollary we have:

Theorem 1.2 (Subgroup GRowth). Let $M$ be as in the Main Theorem. Then for infinitely many $d, f(d)>\exp \left(C d^{\alpha}\right)$.

Strategy of the proof. Step 1. By Theorem 9.1 of [R1], $\pi_{1}(M)$ admits a Zariski dense representation to $S L_{2}(\mathbb{C})$. We use the strong approximation of [We] to find surjective maps from $\pi_{1}(M)$ onto $S L_{2}\left(\mathbb{F}_{q}\right)$, where $\mathbb{F}_{q}$ are residue fields of an algebraic number field $K$.

Step 2. If $\ell$ is a prime, $q, s$ are prime powers such that $\ell$ divides both $\left|S L_{2}\left(\mathbb{F}_{q}\right)\right|$ and $\left|S L_{2}\left(\mathbb{F}_{s}\right)\right|$, and $1 \rightarrow \pi_{1}(N) \rightarrow \pi_{1}(M) \rightarrow S L_{2}\left(\mathbb{F}_{q}\right) \times S L_{2}\left(\mathbb{F}_{s}\right) \rightarrow 1$ is a Galois covering,

${ }^{*}$ Received July 10, 1997; accepted for publication (in revised form) February 6, 1998.

† Department of Mathematical Sciences, University of Durham, Durham DH1 3LE, England (reznikov@mpim-bonn.mpg.de, reznikov@daphne.polytechnique.fr).

$\ddagger$ Max-Planck-Institut für Mathematik, Gottfried-Claren-Str. 26, 53225 Bonn, Germany (moree @mpim-bonn.mpg.de). 
then $H_{1}(N)_{(\ell)}$, the $\ell$ torsion part of $H_{1}(N)$, is nontrivial. This is proved in Proposition 2.1. Moreover, the action of $S L_{2}\left(\mathbb{F}_{q}\right)$ in $H_{1}(N)_{(\ell)}$ is nontrivial (Proposition 2.2).

Step 3. Using Theorem 3.2 it follows that for appropriate $\ell, q$ the $\ell$ rank of $H_{1}(N)_{(\ell)}$ must be $\sim p$, where $q$ is a power of $p$.

It may in principle happen, that just one surjective map $\pi_{1}(M) \stackrel{\alpha}{\longrightarrow} S L_{2}\left(\mathbb{F}_{q}\right)$ is not enough to produce nontrivial $\ell$ homology in $N$, where $\pi_{1}(N)=\operatorname{Ker} \alpha$ (see Step 2 above). We will prove that if this phenomenon happens for infinitely many $p$, then $M$ is hyperbolic in a weak sense (the Gromov simplicial volume is positive).

For a number field $K$, we denote $\mathcal{O}$ its ring of integers, and for a finite set $S$ of primes we denote $\mathcal{O}_{S}$ its localisation at $S$.

THEOREM 1.3 (WEAK HYPERBOLIZATION). Let $M$ be atoroidal. Let $\rho: \pi_{1}(M) \rightarrow$ $S L_{2}\left(\mathcal{O}_{S}\right)$ be a Zariski dense representation. Suppose that for infinitely many primes $\ell$, there exists a rational prime $p \equiv \pm 1(\bmod \ell)$ and a prime ideal $\mathfrak{p} \subset \mathcal{O}$ over $p$ with residue field $\mathbb{F}_{q}$, such that the covering $N$ defined by $1 \rightarrow \pi_{1}(N) \rightarrow \pi_{1}(M) \rightarrow$ $S L_{2}\left(\mathbb{F}_{q}\right) \rightarrow 1$ has trivial $\ell$ homology. Then $M$ has positive Gromov invariant.

REMARK. It is enough to demand that $\ell \nmid \mid H_{3}\left(\left.S L_{2}\left(\mathcal{O}_{s}\right)\right|_{\text {tors }}\right.$, so given the field $K$, the conditions can be effectively checked.

2. Homology of $S L_{2}\left(\mathbb{F}_{q}\right) \times S L_{2}\left(\mathbb{F}_{s}\right)$-coverings. Let $M$ be a closed acyclic 3manifold. In this section, we will study $S L_{2}\left(\mathbb{F}_{q}\right) \times S L_{2}\left(\mathbb{F}_{s}\right)$-coverings of $M$ where $q$ and $s$ are prime powers and $\ell$ divides the orders of $S L_{2}\left(\mathbb{F}_{q}\right)$ and $S L_{2}\left(\mathbb{F}_{s}\right)$, but not $q s$.

Proposition 2.1. Let $1 \rightarrow \pi_{1}(N) \rightarrow \pi_{1}(M) \rightarrow S L_{2}\left(\mathbb{F}_{q}\right) \times S L_{2}\left(\mathbb{F}_{s}\right) \rightarrow 1$ be a Galois covering. Then either $b_{1}(N)>0$, or $\left(H_{1}(N)\right)_{(\ell)} \neq 0$.

Proof. If $N$ is a $\ell$-homology sphere, then the spectral sequence of the covering inplies the direct product $S L_{2}\left(\mathbb{F}_{q}\right) \times S L_{2}\left(\mathbb{F}_{s}\right)$ has periodic $\ell$-cohomology, multiplicatively generated by the Euler class. See [CE]. It follows [CE] that any abelian $\ell$-group in $S L_{2}\left(\mathbb{F}_{q}\right) \times S L_{2}\left(\mathbb{F}_{s}\right)$ should be cyclic, which is obviously wrong.

Consider the tower of coverings $Q \rightarrow N \rightarrow M$, where $1 \rightarrow \pi_{1}(N) \rightarrow \pi_{1}(M) \rightarrow$ $S L_{2}\left(\mathbb{F}_{q}\right) \rightarrow 1$ and $1 \rightarrow \pi_{1}(Q) \rightarrow \pi_{1}(N) \rightarrow S L_{2}\left(\mathbb{F}_{s}\right) \rightarrow 1$ are exact. Suppose $\left(H_{1}(M)\right)_{(\ell)}=0$. Then either $\left(H_{1}(N)\right)_{(\ell)} \neq 0$, or $\left(H_{1}(N)\right)_{(\ell)}=0$ and $\left(H_{1}(Q)\right)_{(\ell)} \neq 0$. Replacing $M$ by $N$ in the latter case, we can assume that the first case holds.

Proposition 2.2. Suppose $1 \rightarrow \pi_{1}(N) \rightarrow \pi_{1}(M) \rightarrow S L_{2}\left(\mathbb{F}_{q}\right) \rightarrow 1$ is a Galois covering of rational homology spheres. Suppose $H_{1}(M)_{(\ell)}=0$ and $H_{1}(N)_{(\ell)} \neq 0$. Then the natural action of $S L_{2}\left(\mathbb{F}_{q}\right)$ in $H^{1}\left(N, \mathbb{F}_{\ell}\right)$ is nontrivial.

Proof. By Quillen $[\mathrm{Qu}]$, the cohomology ring $H^{*}\left(S L_{2}\left(\mathbb{F}_{q}\right), \mathbb{Z}\right)_{\ell}$ is freely generated by one element of degree 4 . Let $W=H^{1}\left(N, \mathbb{F}_{\ell}\right)$, then as an $S L_{2}\left(\mathbb{F}_{q}\right)$-module, $H^{2}\left(N, \mathbb{F}_{\ell}\right) \approx W^{*}$. The spectral sequence of the covering will look like

$$
\begin{aligned}
& \begin{array}{lllllll}
\mathbb{F}_{\ell} & 0 & 0 & \mathbb{F}_{\ell} & \mathbb{F}_{\ell} & \cdots
\end{array} \\
& H^{i}\left(S L_{2}\left(\mathbb{F}_{q}\right), W^{*}\right) \quad \Rightarrow H^{i+j}\left(M, \mathbb{F}_{\ell}\right) \\
& H^{i}\left(S L_{2}\left(\mathbb{F}_{q}\right), W\right)
\end{aligned}
$$$$
\begin{array}{lllllllll}
\mathbb{F}_{\ell} & 0 & 0 & \mathbb{F}_{\ell} & \mathbb{F}_{\ell} & 0 & 0 & \mathbb{F}_{\ell} & \ldots
\end{array}
$$

If the action of $S L_{2}\left(\mathbb{F}_{q}\right)$ in $W$ were trivial, then this would reduce to 


\begin{tabular}{|c|c|c|c|c|c|c|c|}
\hline $\mathbb{F}_{\ell}$ & 0 & 0 & $\mathbb{F}_{\ell}$ & $\mathbb{F}_{\ell}$ & 0 & 0 & \\
\hline$W^{*}$ & 0 & 0 & $\dot{W}^{*}$ & $W^{*}$ & 0 & 0 & $\Rightarrow H^{i+j}\left(M, \mathbb{F}_{\ell}\right)$ \\
\hline$W$ & 0 & 0 & $W$ & $W$ & 0 & 0 & \\
\hline $\mathbb{F}_{\ell}$ & 0 & 0 & $\mathbb{F}_{\ell}$ & $\mathbb{F}_{\ell}$ & 0 & 0 & \\
\hline
\end{tabular}

Then we see that $W^{*}$ which is indexed by $(4 k+3,2)$ in the $E^{2}$-term is not hit by any differential and survives in $E^{\infty}$. This contradicts the finite-dimensionality of $H^{*}(M)$.

3. A variant of Artin's primitive root conjecture. In 1927 Artin conjectured that if $a \neq-1$ or a square, then $a$ is a primitive root $\bmod p$ for infinitely many primes $p$ or, in other words, $\langle a\rangle \cong \mathbb{F}_{p}^{*}$ for infinitely many primes $p$. Under the assumption that the Riemann Hypothesis holds for certain number fields, a quantitative version of the conjecture was proved by Hooley [Ho]. The best known unconditional result to date is due to Heath-Brown [HB]. His main result has the following theorem as a corollary:

THEOREM 3.1. Let $q, r$ and $s$ be three distinct primes. Then at least one of them is a primitive root for infinitely many primes.

In the proof of the Main Theorem we will use the following variant of HeathBrown's result:

THEOREM 3.2. Let $q, r, s$ be three distinct primes each congruent to $3(\bmod 4)$. Then for at least one of them, say $q$, there are infinitely many primes $p$ such that $q$ is a primitive root mod $p$ and, moreover, $p \equiv \pm 1(\bmod q)$. Furthermore, the estimate $\left|\left\{p \leq x:\langle q\rangle \cong \mathbb{F}_{p}^{*}, p \equiv-1(\bmod q)\right\}\right| \gg x(\log x)^{-2}$ holds true.

(Notice that if $\ell \equiv 1(\bmod 4)$ with $\ell$ a prime, then, by quadratic reciprocity, there are no primes $p$ such that $p \equiv \pm 1(\bmod \ell)$ and $\left\langle\ell>\cong \mathbb{F}_{p}^{*}\right.$.)

Proof of Theorem 3.2. Let $q, r, s$ be nonzero integers which are multiplicatively independent. Suppose none of $q, r, s,-3 q r,-3 q s, q r s$ is a square. Suppose, moreover, there exists a prime $p_{0}$ such that

$$
\left(\frac{-3}{p_{0}}\right)=\left(\frac{q}{p_{0}}\right)=\left(\frac{r}{p_{0}}\right)=\left(\frac{s}{p_{0}}\right)=-1 \text { and }\left(p_{0}-1,16 q r s\right) \mid 8 .
$$

Then it follows from the proof of Theorem 1 of [HB] that $N_{q, r, s}^{\prime}(x)$, the number of primes $p \leq x$ for which at least one of $q, r, s$ is a primitive root and such that, moreover, $\bar{p} \equiv p_{0}(\bmod 16 q r s)$, satisfies $N_{q, r, s}^{\prime}(x) \gg x(\log x)^{-2}$.

Now let $q, r$ and $s$ be three distinct primes $\equiv 3(\bmod 4)$. Then none of the integers $q, r, s,-3 q r,-3 q s$ and $q r s$ is a square. We are done if we can find a prime $p_{0}$ such that $p_{0} \equiv-1(\bmod q r s)$ and such that, moreover, $p_{0}$ satisfies $(2)$. Using quadratic reciprocity we see that any prime $p_{0}$ satisfying $p_{0} \equiv 2(\bmod 3), p_{0} \equiv 1(\bmod 4)$, $p_{0} \not \equiv 1(\bmod 16)$ and $p_{0} \equiv-1(\bmod q r s)($ there are actually infinitely many of them), will meet the demands.

The conjecture alluded to in the heading of this section, is the conjecture that if $\ell \not \equiv 1(\bmod 4), \ell$ a prime, then there are infinitely many primes $p$ such that $p \equiv \pm 1$ $(\bmod \ell)$ and $\left\langle\ell>\cong \mathbb{F}_{p}^{*}\right.$. On the generalized Riemann hypothesis this can be shown to be true, and moreover a quantitative version can be established [Mo].

4. Proof of the Main Theorem. By Theorem 9.1 of [R1], there is a Zariski dense representation of $\pi_{1}(M)$ in $S L_{2}(\overline{\mathbb{Q}})$. Let $K$ be the splitting field of this representation, and let $n=[K: \mathbb{Q}]$. By [We], for almost all rational primes $p$ the 
reduction modulo any prime over $p$ in $K$ will define a surjective map $\pi_{1}(M) \rightarrow$ $S L_{2}\left(\mathbb{F}_{q}\right), q=p^{m}, m \leq n$, and moreover, for two such primes $p, f$ the map $\pi_{1}(M) \rightarrow$ $S L_{2}\left(\mathbb{F}_{q}\right) \times S L_{2}\left(\mathbb{F}_{s}\right), q=p^{m}, s=f^{r}$, is surjective. From now on we only look at primes congruent to -1 modulo $\ell$. Suppose that the $\ell$-part of the homology of one such $S L_{2}\left(\mathbb{F}_{s}\right)$-covering $N$ is zero. If this happens for $\ell$ big enough, this alone has far reaching consequences for the nature of $M$ (the Gromov invariant is positive), as we will see in the proof of Theorem 1.3. Now we just notice that, by Proposition 2.1 , we can relabel $N$ by $M$ and assume that for the rest of the primes $p$, either the $\ell$-part of the homology of the $S L_{2}\left(\mathbb{F}_{q}\right)$-covering is nontrivial, or these coverings have positive $b_{1}$. In the first case, by Proposition 2.2 , the action of $S L_{2}\left(\mathbb{F}_{q}\right)$ in $H^{1}\left(N, \mathbb{F}_{\ell}\right)$ is nontrivial. Since $P S L_{2}\left(\mathbb{F}_{q}\right)$ is simple, any element of order $p$ in $S L_{2}\left(\mathbb{F}_{q}\right)$ also acts nontrivially. If $m=\operatorname{dim} H^{1}\left(N, \mathbb{F}_{\ell}\right)$, then we see that $p$ divides $\left|G L_{m}\left(\mathbb{F}_{\ell}\right)\right|$, so that $p \mid(\ell-1)\left(\ell^{2}-1\right) \cdots\left(\ell^{m-1}-1\right)$. By Theorem 3.2 for appropriate $\ell$, there are infinitely many primes $p$ such that the order of $\ell$ in $\mathbb{F}_{p}^{*}$ equals $p-1$. It follows that $m \geq p$. On the other hand, $\left|S L_{2}\left(\mathbb{F}_{q}\right)\right| \sim q^{3}$ and $n=\log _{p} q$ is bounded above by the degree of the number field, over which the representation of $\pi_{1}(M)$ is defined. Finally, $m>$ const $\cdot\left|S L_{2}\left(\mathbb{F}_{q}\right)\right|^{\alpha}$, where $1 / 3 \alpha$ is the degree of the splitting field. The proof is complete in this case. In the other case, we get infinitely many $S L_{2}\left(\mathbb{F}_{q}\right)$-coverings with $b_{1}(N)>0$. Since $b_{1}(M)=0$, the representation of $S L_{2}\left(\mathbb{F}_{q}\right)$ in $H_{1}(N, \mathbb{C})$ does not have a trivial constituent. However, the smallest nontrivial irreducible representation of $S L_{2}\left(\mathbb{F}_{q}\right)$ has dimension $\sim q$, so $b_{1}(N)>d^{1 / 3}$.

Proof of Theorem 1.2. Let $N$ be as above and $m=\operatorname{rank}_{\ell}\left(H_{1}(N)\right)>C d^{\alpha}$. There are at least $\ell^{m-1}$ subgroups of index $\ell$ in $H_{1}(N)_{(\ell)}$. So there are at least $\ell^{C d^{\alpha}-1}$ subgroups of index $\ell d$ in $\pi_{1}(M)$.

Proof of Theorem 1.3. Suppose the Gromov invariant of $M$ is zero. By Proposition 5.4 of [R2], for representation $\sigma: \pi_{1}(M) \rightarrow S L_{2}(K)$, the homology class $\sigma_{*}[M] \in H_{3}\left(S L_{2}(K), \mathbb{Z}\right)$ is torsion. This applies to the representation $\rho: \pi_{1}(M) \rightarrow$ $S L_{2}\left(\mathcal{O}_{S}\right)$. Since the real cohomology of $S L_{2}\left(\mathcal{O}_{S}\right)$ and $S L_{2}(K)$ are isomorphic, $\rho_{*}[M] \in$ $H_{3}\left(S L_{2}\left(\mathcal{O}_{S}\right)\right)$ is also torsion. Now, the $H_{i}\left(S L_{2}\left(\mathcal{O}_{S}\right)\right)$ are finitely generated [BS], so for some $0 \neq N \in \mathbb{Z}$, we have $N \cdot \rho_{*}[M]=0$. From now on we assume that $\ell$ does not divide $N$. Then $\rho_{*}[M]_{(\ell)} \in\left(H_{3}\left(S L_{2}\left(\mathcal{O}_{S}\right)\right)_{\text {tors }}\right)_{(\ell)}=0$. For any surjective homomorphism $S L_{2}\left(\mathcal{O}_{S}\right) \stackrel{\beta}{\longrightarrow} S L_{2}\left(\mathbb{F}_{q}\right)$, we will have $0=(\beta \rho)_{*}[M]_{(\ell)} \in H_{3}\left(S L_{2}\left(\mathbb{F}_{q}\right)\right)_{(\ell)}$. On the other hand by Quillen $[\mathrm{Qu}], H_{3}\left(S L_{2}\left(\mathbb{F}_{q}\right)\right)_{(\ell)} \neq 0$ if $\ell \mid p^{2}-1$. Consider the homology spectral sequence of the covering $1 \rightarrow \pi_{1}(N) \rightarrow \pi_{1}(M) \rightarrow S L_{2}\left(\mathbb{F}_{q}\right) \rightarrow 1$ :

$$
\begin{aligned}
& H_{i}\left(S L_{2}\left(\mathbb{F}_{q}\right), \mathbb{Z}\right) \\
& H_{i}\left(S L_{2}\left(\mathbb{F}_{q}\right), H_{2}(N)\right) \\
& H_{i}\left(S L_{2}\left(\mathbb{F}_{q}\right), H_{1}(N)\right) \\
& H_{i}\left(S L_{2}\left(\mathbb{F}_{q}\right), \mathbb{Z}\right)
\end{aligned} \quad \Rightarrow H_{i+j}(M, \mathbb{Z})
$$

Since the map $H_{3}(M, \mathbb{Z}) \rightarrow H_{3}\left(S L_{2}\left(\mathbb{F}_{q}\right), \mathbb{Z}\right)$ is zero, one of the two differentials $d_{2}$ : $H_{3}\left(S L_{2}\left(\mathbb{F}_{q}\right), \mathbb{Z}\right)_{(\ell)} \rightarrow H_{1}\left(S L_{2}\left(\mathbb{F}_{q}\right), H_{1}(N)\right)_{(\ell)}, d_{3}: \operatorname{Ker} d_{2} \rightarrow H_{0}\left(S L_{2}\left(\mathbb{F}_{q}\right), H_{2}(N)\right)_{(\ell)}$ is nonzero. But if $H_{2}(N) \neq 0$ then $N$ is hyperbolic [Th] and the Gromov invariant of $M$ is positive. If $H_{2}(N)=0$, then $d_{2} \neq 0$, so $H_{1}(N)_{(\ell)} \neq 0$.

Concluding remarks. Theorem 1.3 can be stated with reference made only to representations of $\pi_{1}(M)$ over finite fields:

THEOREM 1.4. Let $M$ be atoroidal.Suppose for infinitely many rational primes $l$, there exists a rational prime $p \equiv \pm 1(\bmod l)$ and a surjective representation $\rho_{l}$ : $\pi_{1}(M) \rightarrow S L_{2}\left(\mathbb{F}_{q}\right)$, where $q$ is a power of $p$, such that the covering defined by $1 \rightarrow$ 
$\pi_{1}(N) \rightarrow \pi_{1}(M) \rightarrow S L_{2}\left(\mathbb{F}_{q}\right) \rightarrow 1$ has trivial l-homology.Then $M$ has positive Gromov invariant.

Proof. Let $F$ be an ultrafilter product of $\mathbb{F}_{q}$, so $\operatorname{char}(F)=0$. Let $\rho: \pi_{1}(M) \rightarrow$ $S L_{2}(\mathbb{F})$ be the ultrafilter product of $\rho_{l}$. Fix an isomorphism between the ultrafilter product of $\vec{F}_{q}$ and $\mathbb{C}$, so $F$ is a subfield of $\mathbb{C}$. If $\rho$ is not rigid as a representation to $S L_{2}(\mathbb{C})$, then $M$ is Haken, therefore hyperbolic.So we may assume $\rho$ is rigid, therefore after a conjugation is defined over a number field $K$. In particular $\left[\mathbb{F}_{q}: \mathbb{F}_{p}\right]$ are bounded. Let $\bar{\rho}$ be the representation defined over $K$ which is conjugate to $\rho$. Then $\bar{\rho}$ is defined over $\mathcal{O}(K)$ since otherwise $M$ is Haken again. Since $\operatorname{Tr}(\bar{\rho})=\operatorname{Tr} \rho$, the reductions of $\bar{\rho}$ are conjugate to $\rho_{l}$ over a quadratic extension of $\mathbb{Q}$. Then the proof goes as in the Theorem 1.3.

\section{REFERENCES}

[BS] A.Borel, J.-P.Serre, Corners and arithmetic groups, Comm. Math. Helv. 48 (1973), pp. $436-491$.

[CE] A.Cartan, S.Eilenberg, Homological Algebra, Princeton University Press, 1956.

[HB] R.Heath-Brown, A remark on Artin's conjecture, Quart. J. Math. Oxford 37 (1986), pp. 2738.

[Ho] C.Hooley, Artin's conjecture for primitive roots, J. Reine Angew. Math. 225 (1967), pp. 209220.

[Mo] P.MoReE, On an conjecture stronger than Artin's primitive root conjecture, unpublished manuscript, 1996.

[Qu] D.QUILlen, On the cohomology and K-theory of general linear group over finite fields, Ann. Math. 96 (1972), pp. 552-586.

[R1] A.REZniKov, Three-manifolds class field theory (Homology of coverings for a nonvirtually $b_{1}$-positive manifold), Selecta Math. 3 (1997), pp. 361-399.

[R2] A.Reznikov, Rationality of secondary classes, Journ. Diff. Geom. 43 (1996), pp. 674-692.

[SW] P.ShALEN, P.WAGREICH, Growth rates, $\mathbb{Z}_{p}$-homology, and volumes of hyperbolic 3-manifolds, Trans. Amer. Math. Soc. 331(1992), pp. 895-917..

[Sh] A.Shalev, Personal communication.

[Th] W.Thurston, Three-dimensional manifolds, Kleinian groups and hyperbolic geometry, Bull. Amer. Math. Soc. 6 (1982), pp. 357-382.

[Tu] V.TURAEV, Nilpotent homotopy type of closed 3-manifolds, LNM 1060 (1984).

[We] B.WEISFELLER, Strong approximation for Zariski-dense subgroups of semi-simple algebraic groups, Ann.Math. 120 (1984), pp. 271-315. 